1 COMPARISON OF RIGHT VENTRICULAR (RV) SIZE AND FUNCTION BY TWO DIMENSIONAL ECHOCARDIOGRAPHY (2DTTE) AND CARDIAC MAGNETIC RESONANCE IMAGING (CMR)

S Akbar*, R Parasa, J Alexander, E Lee, H Kamaruddin, T Mathew. Trent Cardiac Centre, Nottingham University Hospital, Nottingham, UK

10.1136/heartjnl-2015-307845.1

The aim of this study was to compare the right ventricular diameter (RVD1) and tricuspid annular plane systolic excursion (TAPSE) measured by 2DTTE with volumes and EF derived from CMR.

Method and results We studied 50 patients (mean age $53 \pm 17$ years, 36 males and 14 females) who underwent TTE and CMR within 6 months of each other. RVD1 and TAPSE were measured by 3 independent observers (inter observer variability = 5.6\% (95\% CI: 4-6.8\%). RV volumes were measured by 2 independent observers blinded to the TTE data (inter observer variability $=5 \%$ (95\% CI: 4-6.8\%). For RVD1, an upper reference value of $4.2 \mathrm{~cm}$ was used to indicate RV dilatation and for TAPSE a lower reference value of $1.6 \mathrm{~cm}$ was used for systolic impairment as per current recommendations. Upper reference value for end diastolic volume (EDV) indexed to BSA, age and gender was used as the CMR cut off for RV dilatation and lower reference value for EF based on age and gender was used for systolic impairment.

RVD1 showed a statistically significant correlation with $\operatorname{RVEDV}(\mathrm{r}=0.475, \mathrm{p}<0.01)$. The sensitivity and specificity of RVD1 $>4.2 \mathrm{~cm}$ for predicting RV dilatation was $57 \%$ and $83 \%$ respectively. A cut off value of RVD1 $>4.1 \mathrm{~cm}$ improved the sensitivity to $71 \%$ with no change in specificity (ROC analysis: AUC $=0.81)$. TAPSE did not correlate with EF $(r=0.174$, $\mathrm{p}=0.248)$. The sensitivity and specificity of TAPSE $<1.6 \mathrm{~cm}$ for predicting systolic impairment was $81 \%$ and $13 \%$ respectively (ROC analysis: AUC 0.43). Adjusting the cut off of TAPSE did not improve this.

Conclusions Our data suggests that the current value of RVD1 $>4.2 \mathrm{~cm}$ is a poor predictor of RV dilation and lowering the value to 4.1 increases the sensitivity. TAPSE does not correlate with EF by CMR and is a poor predictor of global RV systolic impairment at any cut off value.

\section{ROLE OF CARDIAC MAGNETIC RESONANCE (CMR) IN THE IDENTIFICATION OF ARRHYTHMOGENIC RIGHT VENTRICULAR CARDIOMYOPATHY/DYSPLASIA (ARVC/D) PHENOCOPIES}

\begin{abstract}
${ }^{1,2} \mathrm{AM}$ Amadu, ${ }^{1,3} \mathrm{~A}$ Baritussio, ${ }^{1} \mathrm{~A}$ Ghosh Dastidar, ${ }^{1} \mathrm{~J}$ Rodrigues, ' $\mathrm{M}$ Hamilton, ${ }^{1} \mathrm{~N}$ Manghat, ${ }^{1} \mathrm{C}$ Bucciarelli-Ducci. ${ }^{1}$ Bristol Heart Institute, Bristol NIHR Cardiovascular Biomedical Research Unit (BRU), Bristol, UK; ${ }^{2}$ Department of Radiology, University of Sassari, Sassari, Italy; ${ }^{3}$ Department of Cardiac, Thoracic and Vascular Sciences, University of Padua, Padua, Italy
\end{abstract}

\subsection{6/heartjnl-2015-307845.2}

Introduction ARVC/D is an autosomal dominant genetic heart disease. As part of the diagnostic tools included in the 2010 diagnostic Task Force Criteria, patients are often referred to CMR to rule out the diagnosis. However, the diagnosis is often challenging due to pathologies mimicking ARVC/D.

Aim To assess the prevalence of ARVC/D phenocopies in patients referred to CMR for suspected ARVC/D.

Methods We retrospectively analysed the registry data of patients with suspected ARVC/D referred to CMR in a large UK tertiary centre from January to December 2014. We identified 125 patients (56\% male, median age 40 years) with suspected ARVC/D on the basis of symptoms and clinical presentation, family history of ARVC/D or sudden cardiac death, abnormal electrocardiogram or transthoracic echocardiography. A comprehensive CMR protocol (including cine and late gadolinium enhancement sequences) was performed in all patients.

Results ARVC/D phenocopies were identified in 12 patients (9.6\%): 5 patients had ischaemic heart disease and 7 had nonischaemic heart disease (Table 1). In the latter group, congenital absence of pericardium (Figure 1), idiopathic dilated cardiomyopathy, left ventricular non compaction, arrhythmogenic left ventricular cardiomyopathy (ARVC/D variant), anomalous venous return, atrial septal defect with left to right shunting, and asymmetric pectus excavatum distorting right ventricular (RV) morphology were identified.

Conclusions CMR shows a remarkable role, both in diagnosing ARVC/D and ruling out its mimics. Correct diagnosis of the underlying pathology in patients with suspected ARVC/D is fundamental, given the non-negligible prevalence of phenocopies $(9.6 \%$ in our population) and its subsequent impact on clinical management.

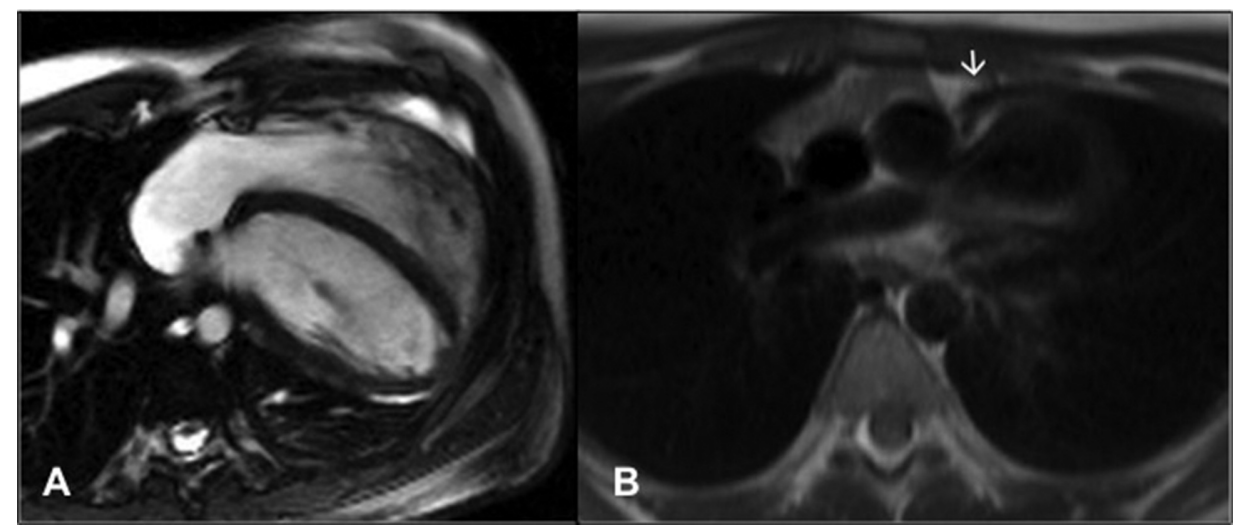

Abstract 2 Figure 1 Typical features of congenital absence of pericardium: heart displacement within the chest, with distorted RV morphology (A), and left lung interposition between the aorta and the pulmonary artery $(B$, arrow). 


\begin{tabular}{lll} 
Abstract 2 Table 1 & ARVC/D phenocopies identified by CMR \\
\hline & ARVC/D Phenocopies ( $\mathrm{n}=12,9.6 \%)$ \\
\cline { 2 - 3 } Patients & Ischaemic Heart Disease & Non-ischaemic Heart Disease \\
\hline Patient A & $\checkmark$ & Congenital absence of pericardium \\
Patient B & & Idiopathic dilated cardiomyopathy \\
Patient C & Left ventricular non-compaction \\
Patient D & $\checkmark$ & Arrhythmogenic left ventricular cardiomyopathy \\
Patient E & Anomalous venous return \\
Patient F & \\
Patient G & \\
Patient H & $\checkmark$ & \\
Patient I & $\checkmark$ & Atrial septal defect \\
Patient L & Asymmetric pectus excavatum \\
Patient M & \\
Patient N & $\checkmark$
\end{tabular}

3 ROLE OF CARDIAC MAGNETIC RESONANCE IN NON-TRAUMATIC OUT OF HOSPITAL CARDIAC ARREST SURVIVORS: A MULTI-CENTRE STUDY

\begin{abstract}
${ }^{1,2} \mathrm{~A}$ Baritussio, ${ }^{2} \mathrm{M}$ Perazzolo Marra, ${ }^{1} \mathrm{~N}$ Ahmed, ${ }^{1} \mathrm{~A}$ Ghosh Dastidar, ${ }^{1} \mathrm{~J}$ Rodrigues, ${ }^{2} \mathrm{~A}$ Zorzi, ${ }^{2} \mathrm{~A}$ Susana, ${ }^{2} \mathrm{D}$ Corrado, ${ }^{1} \mathrm{C}$ Bucciarelli-Ducci. ${ }^{1}$ Bristol Heart Institute, Bristol NIHR Cardiovascular Biomedical Research Unit (BRU), Bristol, UK; ${ }^{2}$ Department of Cardiac, Thoracic and Vascular Sciences, University of Padua, Padua, Italy
\end{abstract}

\subsection{6/heartjnl-2015-307845.3}

Background Non-traumatic out of hospital cardiac arrest (OHCA) is the leading cause of death in Europe and USA. Acute coronary syndromes represent the most common aetiology and in $2 / 3$ of patients a culprit lesion can be identified on angiogram. Among patients with unobstructed coronaries, the differential diagnosis remains unclear. Cardiovascular Magnetic Resonance (CMR), with its superior tissue characterisation, can establish differential diagnosis over and above echocardiography.

Methods This retrospective observational study was performed in two tertiary centres on consecutive patients surviving OHCA referred for CMR (October 2009-January 2015). Coronary angiography and echocardiography were performed as part of the diagnostic work-up. A comprehensive CMR protocol (cine, late gadolinium enhancement, T2 weighted STIR imaging or adenosine perfusion) was performed within 6 weeks from index event.

Results We identified 140 patients (109 male, age $56.1 \pm 15.7$ years) surviving OHCA. 134 patients underwent coronary angiography, with evidence of coronary artery disease (CAD) in
71 (53\%). Sixty-three (47\%) showed unobstructed coronaries. Among patients with CAD, sixty $(85 \%)$ had ischaemic cardiomyopathy on CMR. Among patients with unobstructed coronaries, CMR identified a normal scan in 17 (27\%), ischaemic cardiomyopathy in 9 (14\%), non-ischaemic cardiomyopathy in 27 (43\%) (Figure 1), and non-specific findings in 10 (16\%). Different underlying cardiomyopathies have been identified by CMR among patients with non-ischaemic cardiomyopathy, as shown in Table 1. Overall, the diagnostic pick-up rate of CMR was $91 \%$.

Conclusion Cardiac MRI identified the underlying diagnosis in the large majority of patients, particularly in those with unobstructed coronaries (pick-up rate 84\%), leading to a change in management in all. CMR has therefore a promising role in the clinical work-up of patients surviving OHCA.

Abstract 3 Table 1 CMR findings among patients with non-ischaemic cardiomyopathy

\begin{tabular}{ll}
\hline CMR diagnosis & $\mathbf{n = 2 7}$ \\
\hline Dilated cardiomyopathy & 6 \\
Hypertrophic cardiomyopathy & 3 \\
Myocarditis & 7 \\
Tako-Tsubo cardiomyopathy & 2 \\
Mitral valve prolapse & 4 \\
Hypertensive heart disease & 1 \\
Cardiac amyloid & 1 \\
Left ventricular non compaction & 1 \\
Biventricular arrhythmogenic cardiomyopathy & 1 \\
Heart failure with preserved ejection fraction & 1 \\
\hline
\end{tabular}

\section{$4 \quad$ EXTRACELLULAR VOLUME IN THE INFARCT ZONE IS ASSOCIATED WITH CLINICAL AND MRI MEASURES OF INFARCT SEVERITY IN SURVIVORS OF ACUTE STEMI}

${ }^{1} \mathrm{~J}$ Carberry ${ }^{*},{ }^{1,2} \mathrm{D}$ Carrick, ${ }^{3} \mathrm{C}$ Haig, ${ }^{1} \mathrm{SM}$ Rauhalammi, ${ }^{1} \mathrm{~N}$ Ahmed, ${ }^{1,2} \mathrm{I}$ Mordi, ${ }^{2} \mathrm{M}$ McEntegart, ${ }^{1} \mathrm{M}$ Petrie, ${ }^{1} \mathrm{H}$ Eteiba, ${ }^{1} \mathrm{~S}$ Hood, ${ }^{1} \mathrm{~S}$ Watkins, ${ }^{1} \mathrm{M}$ Lindsay, ${ }^{1} \mathrm{~A}$ Davie, ${ }^{2} \mathrm{~A}$ Mahrous, ${ }^{1} \mathrm{~A}$ Radjenovic, ${ }^{3}$ I Ford, ${ }^{1} \mathrm{KG}$ Oldroyd, ${ }^{1,2} \mathrm{C}$ Berry. ${ }^{1} \mathrm{BHF}$ Glasgow Cardiovascular Research Center, Institute of Cardiovascular and Medical Sciences, University of Glasgow, Glasgow, UK; ${ }^{2}$ West of Scotland Heart and Lung Center, Golden Jubilee National Hospital, Dumbartonshire, UK; ${ }^{3}$ Robertson Center for Biostatistics, University of Glasgow, Glasgow, UK

\subsection{6/heartjnl-2015-307845.4}

Background The clinical significance of extracellular volume (ECV) expansion in infarcted myocardium post-STEMI is unknown. Myocardial ECV can be estimated by cardiac magnetic

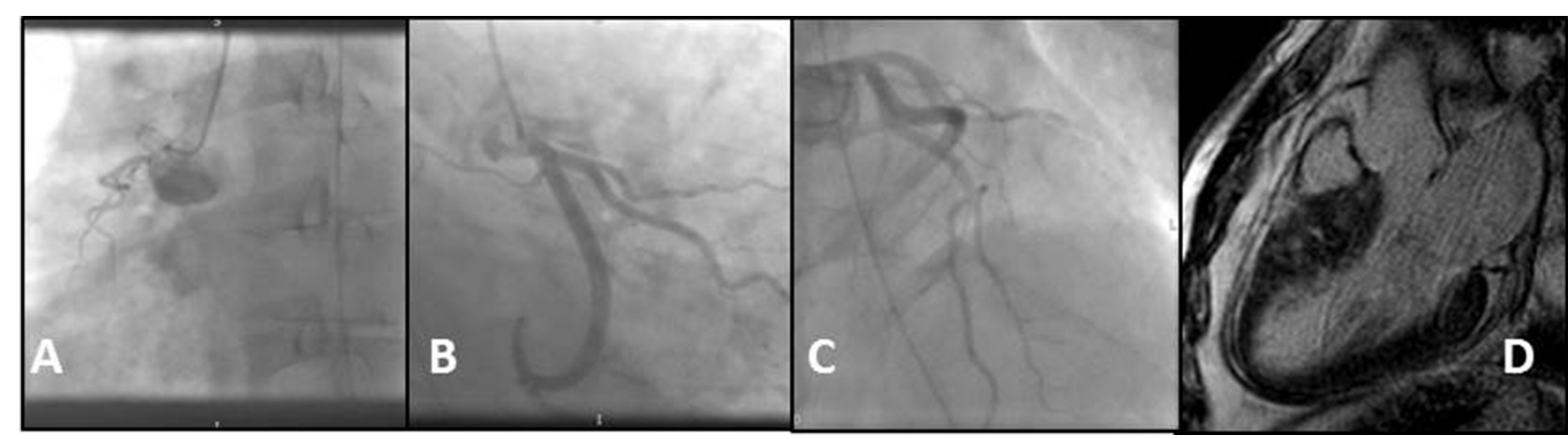

Abstract 3 Figure 1 Unobstructed coronaries $(A, B, C)$ in a patient with hypertrophic cardiomyopathy with patchy septal myocardial late enhancement (D). 